\title{
SOME PROPERTIES OF THE FEYNMAN-KAC FUNCTIONAL
}

\author{
S. GRAVERSEN \\ Matematisk Unstitut, Aarhus University \\ Aarhus, Denmark \\ Z.R. POP-STOJANOVIĆ \\ and \\ K. MURALI RAO \\ Department of Mathematics, University of Florida \\ Gainesville, Florida 32611 USA
}

(Received May, 1994; revised December, 1994)

\begin{abstract}
The Feynman-Kac formula and its connections with classical analysis were initiated in the now celebrated paper [6] of M. Kac. It soon became obvious that the formula provides a powerful tool for solving partial differential equations by running the Brownian motion process. K.L. Chung and K.M. Rao in [4] used it to characterize solutions of the Schrödinger equation. In this paper we study some properties of the Feynman-Kac functional using the Brownian motion process. In particular, we are going to use it in connection with the gauge function in order to obtain an energy formula similar to one obtained by G. Dal Maso and U. Mosco in [5].
\end{abstract}

Key words: Gauge Function, Brownian Motion, Subharmonic Function, Explosion Point, Kato Class, the Energy Functional, Excessive Function.

AMS subject classification: $60 \mathrm{~J} 45,60 \mathrm{~J} 25,35 \mathrm{C} 05$.

\section{Introduction}

In [5], G. Dal Maso and U. Mosco studied a relaxed Dirichlet problem in an open region $\Omega$ of $R^{d}, d \geq 2$, which can formally be written as:

$$
-\Delta u+\mu u=0 \text { in } \Omega
$$

where $\Delta$ is the Laplace operator and $\mu$ is an arbitrary non-negative Borel measure not charging polar sets in $R^{d}$. The measure $\mu$ may take the value $+\infty$. Special cases of (1.1) are Dirichlet problems of the type

$$
-\Delta u=0 \text { in } \Omega-\bar{E}, \quad u=0 \text { on } E
$$

$(\bar{E}$ denotes the closure of $E)$, as well as the stationary Schrödinger equation:

$$
-\Delta u+q(x) u=0 \text { in } \Omega
$$


where $q$ is a non-negative potential. The main result in [5] is an approximation of equation (1.1) by a sequence of Dirichlet problems of the form (1.2). In order to carry out this procedure, one needs to study the behavior of an arbitrary local weak solution $u$ of (1.1) such that $u \in$ $\mathcal{H}_{l o c}^{1}(\Omega) \cap L_{l o c}^{2}(\Omega, \mu)$ and having finite local $\mu$-energy given by:

$$
\int_{\Omega^{\prime}}|\nabla u|^{2} d x+\int_{\Omega^{\prime}} u^{2} d \mu, \quad \Omega^{\prime} \subset \Omega .
$$

The methods in [5] are variational in nature. In this paper, we study the Feynman-Kac functional, directly using the Brownian motion process. Using the gauge function, we obtain an energy formula in part 5 similar to formula (1.3). Section 3 deals with the characterization of the null set of $g_{q}$. For example, in Proposition 3.3, it is shown that the set $\left\{g_{q}=0\right\}$ is a polar set. Section 4 deals with continuity properties of the gauge function $g_{q}$. Specifically, Theorem 4.2 shows that if $g_{q}$ is nonvanishing and continuous in $\Omega$, then $q$ is in local Kato class in $\Omega$. Finally, Section 5 deals with the energy as introduced in [7]. In addition, it is shown that if $s$ is an excessive function, then its corresponding gauge function satisfies the equation $\Delta g_{s}=\frac{1}{s} g_{s}$.

\section{Notations and Preliminaries}

Throughout this paper, $X=\left\{X_{t} ; t \geq 0\right\}$ denotes the Brownian Motion process in $R^{d}, d \geq 2 ; \Omega$ denotes a domain in $R^{d}$. Let $q \geq 0$ be measurable. Let $e_{q}(t)=\exp \left[-\int_{0}^{t} q\left(X_{s}\right) d s\right], g_{q}(x)=$ $E^{x}\left[e_{q}(\tau)\right]$, where $\tau=$ exit time from $\Omega$. Using strong Markov property, we see that $g_{q} \leq$ $E^{x}\left[g_{q}\left(X_{\tau_{B}}\right)\right]$, where $\tau_{B}=$ exit time from a ball with a center $x$. Also, it is seen that if $q$ is bounded, then $g_{q}$ is continuous; hence, in general, $g_{q}$ is upper semi-continuous. It follows that $g_{q}$ is subharmonic in $\Omega$.

Throughout this paper we deal with a topology on $R^{d}$ that is finer than the Euclidean metric topology. Namely, the fine topology on $R^{d}$ is the smallest topology on $R^{d}$ for which all superharmonic functions are continuous in the extended sense. It is easily seen that the fine topology is larger than the Euclidean metric topology on $R^{d}$. So we speak of fine interior, finely continuous, etc.

Another concept which is used in the paper and which is related to the behavior of the Brownian motion process is that of a regular point of a set. Namely, given a set $D$ denote by $T$ the exit time for the Brownian motion from $D$. (Which is the same as the hitting time of the complement of $D$.) Then, a point $a \in \partial D$ is called regular for $D^{c}$ if $P^{a}\{T=0\}=1$. In other words, starting at a regular point the Brownian motion hits the complement of a set in question immediately.

\section{The Null Set of $g_{q}$}

In this section we study the set on which function $g_{q}$ vanishes.

Proposition 3.1: $q<\infty$ a.e. on the set $\left\{g_{q}>0\right\}$.

Proof: Suppose $q$ is bounded. Then, assuming the domain $\Omega$ is bounded,

$$
1-e_{q}(\tau)=\int_{0}^{\tau} q\left(X_{s}\right) e^{-\int_{s}^{\tau} q\left(X_{\theta}\right) d \theta} d s .
$$


Therefore if $q$ is bounded,

$$
1-g_{q}(x)=G\left[q g_{q}\right]
$$

which is a consequence of (3.1) and Markov property.

For general $q$, let $a_{n}=q \wedge n$. Using (3.2), one gets,

$$
1-g_{q_{n}}(x)=G\left[q_{n}, g_{q_{n}}\right] \geq G\left[q_{n} g_{q}\right] .
$$

Let $n$ tend to infinity in the above inequality. We get

$$
1=g_{q} \geq G\left[q g_{q}\right]
$$

where $q g_{q}$ is defined to be zero if $g_{q}=0$. Thus, $q g_{q}<\infty$ a.e.

A more precise result is the following:

Proposition 3.2: Suppose $g_{q}(\xi)=0$ and that $\xi$ is regular for the set $\left\{g_{q}>0\right\}$. Then, $\xi$ is an "explosion point" for q, i.e.,

$$
\forall t>0, P^{\xi}\left\{\int_{0}^{t} q\left(X_{s}\right) d s=\infty\right\}=1 .
$$

Proof: Let $\varepsilon>0, F=\left\{g_{q} \geq \varepsilon\right\}$ and let $T$ be the hitting time of $F$. Then,

$$
0=g_{q}(\xi) \geq E^{x}\left[e_{q}(T) g_{q}\left(X_{T}\right): T<\tau\right]
$$

implying $e_{q}(T)=0$ on the set $\{T<\tau\}$. As $\varepsilon \downarrow 0$, the hitting times decrease to the hitting time to $\left\{g_{q}>0\right\}$ which is zero $P^{\xi_{-a . s}}$. by assumption. This completes the proof.

Remark 3.1: If $g_{q}(\xi)=0$ and $\xi$ is not regular for $\left\{g_{q}>0\right\}$, then $\xi$ must be in the fine interior of $\left\{g_{q}=0\right\}$. Thus,

$$
\left\{g_{q}=0\right\}=\{\text { finely open set }\} \cup\{\text { the set of explosion points of } q\} \text {. }
$$

Remark 3.2: Suppose $g_{q} \not \equiv 0$. Then $\left\{g_{q}>0\right\}$ is a finely open non-empty set. So, some point of $\left\{g_{q}=0\right\}$ must be a regular point for $\left\{g_{q}>0\right\}$. However, such a point is an explosion point for $q$. Thus, we can say if $q$ has no explosion points, then $g_{q}$ cannot vanish unless $g_{q} \equiv 0$.

Let us show that some point for which $g_{q}=0$ is regular for $\left\{g_{q}>0\right\}$. Let $g_{q}(\xi)=0$ and $T=$ hitting time to $\left\{g_{q}>0\right\}$. Since $g_{q}\left(X_{t}\right)$ is continuous in $t$ for $t>0$, we see that $g_{q}\left(X_{T}\right)=0$ if $T<\infty$ and $X_{T}$ is regular for $\left\{g_{q}>0\right\}$, almost surely. Hence, there are points for which $g_{q}=0$, and which are regular for $\left\{g_{q}>0\right\}$.

Proposition 3.3: Suppose that for some $\xi, P^{\xi}\left\{\int_{0}^{\tau} q\left(X_{s}\right) d s<\infty\right\}=1$. This implies that $\left\{g_{q}=0\right\}$ is a Polar set. Furthermore,

$$
1-g_{q}=G\left(q g_{q}\right) .
$$

Proof: Indeed, $g_{q}$ is finely continuous so that set $A=\left\{g_{q}=0\right\}$ is finely closed. If $T=$ hitting time of $A$, we have

$$
E^{\xi}\left[e^{\left.-\int_{0}^{\tau}: T<\tau\right]=E^{\xi}\left[e^{-\int_{0}^{T}} g_{q}\left(X_{T}\right): T<\tau\right]=0}\right.
$$


because $g_{q}\left(X_{T}\right)=0$ on the set $(T<\tau)$. Thus, $P^{\xi}\left[e^{-\int^{\tau}} 1_{T<\tau}=0\right]=1$. However, it is given that $e^{-\int_{0}^{\tau}}>0$ with $P^{\xi}$-probability 1 . Hence, we conclude that $A$ is a Polar set.

Remark 3.3: The proof of formula (3.3) is as follows.

We have $P^{\xi}\left[\int_{0}^{\tau} q<\infty\right]=1$. Therefore the following arguments are valid: Let $q_{n} \uparrow q$ with $G\left(q_{n}\right)$ bounded. Then we know that (3.3) holds with $q$ replaced by $q_{n}$. By taking limits, we get $G\left(q g_{q}\right) \leq 1-g_{q}$. By definition, we have always $q g_{q}=0$ on the set $\left\{g_{q}=0\right\}$. At $\xi$ we have

$$
\begin{gathered}
G\left(q g_{q}\right)(\xi)=E^{\xi}\left[\int_{0}^{\tau} q\left(X_{s}\right) g_{q}\left(X_{s}\right) d s\right] \\
=E^{\xi}\left[\int_{0}^{\tau} q\left(X_{s}\right) e^{-\int_{s}^{\tau} q\left(X_{u}\right) d u} d s\right] \\
=E^{\xi}\left[1-e^{-\int_{0}^{\tau} q}\right]=1-g_{q}(\xi) .
\end{gathered}
$$

Now we can claim that $1-g_{q}-G\left(q g_{q}\right)$ is excessive. Indeed, if $q_{n} \uparrow q$,

$$
\begin{gathered}
1-g_{q}-G\left(q g_{q}\right)=\lim _{n}\left[1-g_{q_{n}}-G\left(q_{n} g_{q}\right)\right] \\
=\lim _{n}\left[G\left(q_{n} g_{q_{n}}\right)-G\left(q_{n} g_{q}\right)\right] \\
=\lim _{n} G\left[q_{n}\left(g_{q_{n}}-g_{q}\right)\right]
\end{gathered}
$$

and each is excessive. Thus, since (3.3) holds at $\xi$, it holds everywhere. Remark 3.4: Under the assumption that for some $\xi, P^{\xi}\left[\int_{0}^{\tau} q<\infty\right]=1$, we can show that the
only zeros of $g_{q}$ are the explosion points of $q$.

Indeed, if $g_{q}(x)=0$ we have

$$
0=g_{q}(x) \geq E^{x}\left[e^{-\int_{0}^{t}} g_{q}\left(X_{t}\right): t<\tau\right]
$$

Since $g_{q}\left(X_{t}\right)>0, e_{q}(t)=0$ for every $t$, which means that $x$ is an explosion point.

Example: Let $q(x)=|x|^{-\beta}, 2<\beta<3$ in $R^{3}$. Then, using scaling for Brownian motion we see that for any $t>0$,

$$
\begin{gathered}
P^{0}\left\{\int_{0}^{t}\left|X_{s}\right|^{-\beta} d s<a\right\}=P^{0}\left\{\int_{0}^{t}\left|\varepsilon X_{s / \varepsilon^{2}}\right|^{-\beta} d s<a\right\} \\
=P^{0}\left\{\varepsilon^{2-\beta} \int_{0}^{t / \varepsilon^{2}}\left|X_{s}\right|^{-\beta} d s<a\right\} .
\end{gathered}
$$

The last inequality holds for every $\varepsilon>0$. It follows that $\int_{0}^{t}\left|X_{s}\right|^{-\beta} d s \equiv \infty, \forall t>0, P^{0}-$ a.s., i.e., 0 is an explosion point for $q$ and, of course, $g_{q}(0)=0$. Note that $q$ is locally integrable. If 
$\left\{\xi_{i}\right\}$ is a dense countable set, let $q_{i}=\left|x-\xi_{i}\right|^{-\beta}$. Then $q_{i}$ is locally integrable. For suitable constants $\eta_{i}, q=\sum \eta_{i} q_{i} \in L_{l o c}^{1}$, and $\forall t>0$ and $\forall i, P^{\xi_{i}}\left[\int_{0}^{t} q\left(X_{s}\right) d s=\infty\right]=1$. Thus, it is possible that $g_{q}=0$ on a dense set with $q$ integrable.

\section{Continuity Properties of $g_{q}$}

The following remark will be helpful in the proof of Proposition 4.1.

Remark: The function

$$
G(x, t)=P^{x}[\tau<t]+E^{x}\left[g_{q}\left(X_{t}\right): t<\tau\right]
$$

decreases as $t$ decreases and tends to $g_{q}$ as $t$ tends to zero for each fixed $x$.

Indeed, if $s<t$, we may compute

$$
\begin{gathered}
G(x, t)-G(x, s)=P^{x}[s<\tau<t]+E^{x}\left[\left\{\exp \left(-\int_{t}^{\tau} q\left(X_{u}\right) d u\right)-\exp \left(-\int_{s}^{\tau} q\left(X_{u}\right) d u\right)\right\}: t<\tau\right] \\
-E^{x}\left[\exp \left(-\int_{s}^{\tau} q\left(X_{u}\right) d u\right): s<\tau<t\right] \geq 0 .
\end{gathered}
$$

Also, $G(\cdot, t)$ is continuous for each $t$. Thus, if $g_{q}$ is continuous, $G(\cdot, t)$ tends to $g_{q}$ uniformly on compacts by Dini's theorem. We conclude that $g_{q}$ is continuous if and only if $E^{(\cdot)}\left[g_{q}\left(X_{t}\right): t<\tau\right]$ tends to $g_{q}$ uniformly on compacts as $t$ tends to zero.

Proposition 4.1: Let $g_{i}=g_{q_{i}}$ be continuous. Then $g=g_{q_{1}+q_{2}}$ is also continuous.

Proof: If $h$ is any of $g_{1}, g_{2}$ or $g$ and $r$ any of $q_{1}+q_{2}, q_{1}$ or $q_{2}$, we have by Markov property for any $t$,

$$
\begin{gathered}
E^{x}\left[h\left(X_{t}\right): t<\tau\right]-h(x)=E^{x}\left[h\left(X_{t}\right)\left(1-e^{-\int_{0}^{t} r\left(X_{s}\right) d s}\right): t<\tau\right] \\
-E^{x}\left[e^{-\int_{0}^{\tau} r\left(X_{s}\right) d s}: \tau<t\right] .
\end{gathered}
$$

The last term above clearly tends to zero uniformly on compacts because $P^{x}\{\tau<t\}$ does this as $t \rightarrow 0$. From the last sentence of the above remark, the continuity of $h$ is equivalent to

$$
E^{(\cdot)}\left[h\left(X_{t}\right)\left(1-e_{r}(t)\right): t<\tau\right]
$$

to tend to zero uniformly as $t \rightarrow 0$. If $h=g_{i}$ and $r=q_{i}, i=1,2$, this is the case because $g_{i}$ are continuous. We have with $h=g$ and $r=q_{1}+q_{2}$, that

$$
\begin{gathered}
E^{(\cdot)}\left[g\left(X_{t}\right)\left(1-e_{\tau}(t)\right): t<\tau\right] \\
=E^{(\cdot)}\left[g\left(X_{t}\right)\left(1-e_{q_{1}}(t)+e_{q_{1}}(t)-e_{r}(t)\right): t<\tau\right] .
\end{gathered}
$$

Since $g \leq g_{1}$

$$
E^{(\cdot)}\left[g\left(X_{t}\right)\left(1-e_{q_{1}}(t)\right): t<\tau\right] \leq E^{(\cdot)}\left[g_{1}\left(X_{t}\right)\left(1-e_{q_{1}}(t)\right): t<\tau\right]
$$


using the conclusion stated in the last sentence of the previous remark, it follows that the lefthand side of this inequality tends to zero uniformly as $t \downarrow 0$, because the right-hand side does it, since $g_{1}$ is continuous. Likewise,

$$
E^{(\cdot)}\left[g\left(X_{t}\right)\left(e_{q_{1}}(t)-e_{r}(t)\right): t<\tau\right] \leq E^{(\cdot)}\left[g_{2}\left(X_{t}\right)\left(1-e_{q_{2}}(t)\right): t<\tau\right],
$$

where again the left-hand side of this inequality tends to zero uniformly as $t \downarrow 0$, because the righthand side does it, since $g_{2}$ is continuous. This concludes the proof showing that $g_{q_{1}+q_{2}}$ is also continuous.

Proposition 4.2: If $g_{q}$ is continuous, so is $g_{\lambda_{q}}$ for all $\lambda>0$.

Proof: Because of Proposition 4.1, it suffices to prove Proposition 4.2 for $0<\lambda<1$.

As in the proof of Proposition 4.1, we need only to show that if $g_{\lambda}=g_{\lambda_{q}}$,

$$
E^{x}\left[g_{\lambda}\left(X_{t}\right)\left(1-e_{\lambda_{q}}(t)\right): t<\tau\right]
$$

tends to zero uniformly on compacts. By Hölder's inequality, the expression in (4.2) is less than or equal to

$$
\left\{E^{x}\left[g_{\lambda}^{\lambda^{-1}}\left(X_{t}\right)\left(1-e_{\lambda_{q}}(t)\right)^{\lambda-1}: t<\tau\right]\right\}^{\lambda}
$$

Now we use Hölder's inequality again to obtain $g_{\lambda} \leq g_{q}^{\lambda}$ and clearly,

$$
\left(1-e_{\lambda_{q}}(t)\right)^{\lambda-1} \leq 1-e_{\lambda_{q}} \leq 1-e_{q}(t)
$$

Thus, it follows from (4.3) that the expression in (4.2) is less than or equal to

$$
E^{x}\left[g_{q}\left(X_{t}\right)\left(1-e_{q}(t)\right): t<\tau\right]^{\lambda}
$$

and the result follows.

Theorem 4.1: Suppose $G q$ is locally bounded. Then $g_{q}$ is continuous if and only if $G q$ is continuous.

Proof: Step 1. Suppose $G q$ is bounded. Then $g_{q}$ is continuous if and only if $G q$ is continuous. To see this, write

and compute:

$$
K_{q} f=E^{x}\left[\int_{0}^{\tau} e_{q}(t) f\left(X_{t}\right) d t\right]
$$

$$
\begin{gathered}
g_{q}=1-K_{q} q \\
G q=K_{q} q+K_{q}[q G q] .
\end{gathered}
$$

The first equation shows that $g_{q}$ is continuous if and only if $K_{q} q$ is continuous, and from the second equation, we see that under the condition that $G q$ is bounded, the continuity of $G q$ is equivalent to that of $K_{q} q$.

Step 2. Now suppose $D$ is a relatively compact subset of $\Omega$. Then, $G_{D} q \leq G_{\Omega} q$ and $G_{\Omega} q$ bounded on $D$ implies that $G_{D} q$ is bounded. Suppose $g_{q}$ is continuous. We have

$$
g_{q}=E^{x}\left[e_{q}\left(\tau_{D}\right) g_{q}\left(X_{\tau_{D}}\right)\right]
$$


Now by Jensen's inequality, $g_{q}(x) \geq e^{-G q(x)}$, so that $g_{q}$ is bounded from below on compacts, say, $g_{q}\left(X_{\tau_{D}}\right) \geq \varepsilon$. Then, one can write

$$
g_{q}(x)=\varepsilon E^{x}\left[e_{q}\left(\tau_{D}\right)\right]+E^{x}\left[e_{q}\left(\tau_{D}\right)\left(g_{q}\left(X_{\tau_{D}}\right)-\varepsilon\right)\right] .
$$

Both terms on the right-hand side of this equation are upper-semi-continuous functions on $D$. However, the left-hand side is continuous on $D$ implying that the both terms on the right-hand side are continuous. This implies that $E^{x}\left[e_{q}\left(\tau_{D}\right)\right]$ is continuous on $D$. This fact and the result from Step 1, imply that $G_{D} q$ is continuous. However, on $D, G q=G_{D} q+$ a harmonic function. This implies that $G q$ is continuous on $D$.

Suppose now $G q$ is continuous. Then, for each relatively compact open subset $D, G_{D} q$ is bounded and continuous. Hence, from Step $1, E^{(\cdot)}\left[e_{q}\left(\tau_{D}\right)\right]$ is continuous and, from (4.4), so is $g_{q}$. This completes the proof.

Theorem 4.2: Let $g_{q}$ be nonvanishing and continuous in $\Omega$. Then $q$ is in local Kato class in $\Omega$, i.e., $G q 1_{F}$ is continuous and bounded in $\Omega$ for each relatively compact set $F$.

Proof: Step 1. First, suppose that $g_{q}$ is bounded from below and continuous in $\Omega$. Say, $g_{q} \geq \varepsilon$. Then, we claim that $G q$ is bounded and continuous in $\Omega$. To see this, note that

$$
1-g_{q} \geq G\left[q g_{q}\right] \geq \varepsilon G q
$$

giving $G q \leq \frac{1-\varepsilon}{\varepsilon}$, i.e., $G q$ is bounded. Then, by Theorem 4.1, $G q$ is continuous.

Step 2. Again, we localize the problem. Let $D$ be a relatively compact subset of $\Omega$. We have

$$
g_{q}=E^{x}\left[e_{q}\left(\tau_{D}\right) g_{q}\left(X_{\tau_{D}}\right)\right]
$$

Since $g_{q}$ is nonvanishing and continuous, it is bounded from below on $\bar{D}$. So, as in Theorem 4.1, we see that $E^{x}\left[e_{q}\left(\tau_{D}\right)\right]$ is continuous $D$. Moreover, $E^{x}\left[e_{q}\left(\tau_{D}\right)\right] \geq g_{q} \geq \varepsilon$, say, (because $g_{q}$ is bounded from below on compacts), implying from Step 1 that $G_{D} q$ is bounded and continuous on $D$. This completes the proof.

Finally, we have:

Theorem 4.3: Suppose $G q \not \equiv \infty$. If $g_{q}$ is continuous, then $G q$ is extended continuous and $\left\{g_{q}=0\right\}=\{G q=\infty\}$. If $G q$ is extended continuous, then $g_{q}$ is continuous at every point at which $G q(x)<\infty$.

Proof: Suppose $g_{q}$ is continuous. Then the set $D_{0}=\left\{g_{q}>0\right\}$ is an open subset of $\Omega$. Also, the set $\{G q(x)<\infty\}$ is contained in $D_{0}$. Indeed, if $G q(x)<\infty$, then $P^{x}\left\{\int_{0}^{\tau} q\left(X_{s}\right) d s<\infty\right\}=1$, implying that $g_{q}(x)>0$.

We will show that $G q$ is continuous on $D_{0} . G q$ is necessarily continuous at each $x$, such that $G q(x)=\infty$, and this set contains $D_{0}^{c}$. Let $D$ be a relatively compact open subset of $D_{0}$ and set $\tau_{1}=\tau_{D}$. Then,

$$
g_{q}(x)=E^{x}\left[e_{q}\left(\tau_{1}\right) g_{q}\left(X_{\tau_{1}}\right)\right] .
$$

Since $g_{q}$ is continuous and strictly positive on $\bar{D}, E^{x}\left[e_{q}\left(\tau_{1}\right)\right]$ is continuous and bounded from below on $D$ (it dominates $g_{q}$ on $D$ ). Hence, by Theorem 4.1, $G_{D} q$ is continuous and bounded in $D$. Since $G q=G_{D} q+$ a harmonic function in $D$, we see that $G q$ is continuous on $D$. Thus, $G q$ is continuous and finite on $D_{0}$. Suppose now $G q$ is extended continuous. Let $U=\{G q<N\}$. $U$ 
is an open set. We need only show that $g_{q}$ is continuous on $U$. Now, on $U, G_{U} q$ is bounded and continuous. So, from Theorem $4.1, E^{(\cdot)}\left[e_{q}^{q}\left(\tau_{U}\right)\right]$ is continuous. Hence, so is $g_{q}$. This completes the proof.

Lemma 4.1: Let $f_{\lambda}, 0 \leq \lambda \leq 1$, be a family of lower semi-continuous functions on $X$. Suppose $0 \leq f_{\lambda} \leq 1$ and that $f_{\lambda}(x)$ is increasing in $\lambda$. Let

$$
f(x)=\int_{0}^{1} f_{\lambda}(x) d \lambda .
$$

Then, $f$ continuous at $x_{0}$ implies $f_{\lambda}(\cdot)$ is continuous at $x_{0}$ for every $\lambda$ which is a continuity point of $\lambda \rightarrow f_{\lambda}\left(x_{0}\right)$.

Proof: Suppose $f$ is continuous at $x_{0}$ and $\mu$ is a continuity point of $\lambda \rightarrow f_{\lambda}\left(x_{0}\right)$. Since $f_{\lambda}\left(x_{0}\right)$ is increasing in $\lambda$, we see that

$$
\frac{1}{h} \int_{\mu}^{\mu+h} f_{\lambda}(x) d \lambda
$$

is increasing in $h$. Also, function $\int_{\mu}^{\mu+h} f_{\lambda}(x) d \lambda$ and hence, the function in (4.7), is continuous at $x_{0}$. As $h \downarrow 0$ these functions decrease to $f_{\mu}(x)$. Thus, at $x_{0}, f_{\mu}$ is upper semi-continuous function. Since $f_{\mu}$ is lower semi-continuous by assumption, the proof is complete.

Corollary 4.1: $g_{q}$ is continuous if and only if $x \rightarrow P^{x}\left\{A_{\infty}>\lambda\right\}$ is continuous for all $\lambda>0$, where $A_{\infty}=\int_{0}^{\tau} q\left(X_{s}\right) d s$.

Proof: We have

$$
\begin{gathered}
g_{q}(x)=E^{x}\left[e^{\left.-A_{\infty}\right]}=\int_{0}^{1} P^{x}\left\{e^{\left.-A_{\infty}>t\right\} d t}\right.\right. \\
=\int_{0}^{1} P^{x}\left\{\frac{1}{t}>e^{A}\right\} d t=1-\int_{0}^{1} P^{x}\left\{A_{\infty}>\log \frac{1}{t}\right\} d t .
\end{gathered}
$$

The family $f_{t}(x)=P^{x}\left\{A_{\infty}>\log \frac{1}{t}\right\}$ satisfies the conditions of Lemma 4.1. Thus, $x \rightarrow P^{x}\left\{A_{\infty}>\mu\right\}$ is continuous at $x_{0}$ provided $\lambda \rightarrow P^{x} 0\left\{A_{\infty}>\lambda\right\}$ is continuous at $\mu$. It is easy to see that for any $x$, $P^{x}\left\{A_{\infty}=\lambda\right\}=0$. In other words, $\lambda \rightarrow P^{x}\left\{A_{\infty}>\lambda\right\}$ is continuous in $\lambda$ for each $x$. Thus, the continuity of $g$ implies that of $x \rightarrow P^{x}\left\{A_{\infty}>\lambda\right\}$ for each $\lambda>0$. Conversely, if $x \rightarrow P^{x}\left\{A_{\infty}>\lambda\right\}$ is continuous, then so is $x \rightarrow P^{x}\left\{e^{-A_{\infty}}>\lambda\right\}$. Finally, by bounded convergence theorem, it follows that $\int_{0}^{1} P^{x}\left\{e^{-A}>\lambda\right\} d \lambda$ is also continuous.

\section{Miscellaneous Results}

Some simple facts: The following inequalities will be used in the sequel.

$$
e^{-a}+e^{-b} \geq 1+e^{-a-b}, a, b \geq 0,
$$

so that if $c \leq a \wedge b$,

$$
e^{-(a-c)}+e^{-(b-c)} \leq 1+e^{-(a-c)-(b-c)}
$$


or

$$
e^{-a}+e^{-b} \leq e^{-c}+e^{-a-b+c}, a, b \geq 0, c \leq a \wedge b
$$

Thus, if $q_{1}, q_{2} \geq 0$ and $a=\int_{0}^{t} q_{1}, b=\int_{0}^{t} q_{2}, c=\int_{0}^{t}\left(q_{1} \wedge q_{2}\right)\left(X_{s}\right) d s$ we get from (5.2) since $a+b-$ $c=\int_{0}^{t} q_{1} \vee q_{2}$

$$
e_{q_{1}}(t)+e_{q_{2}}(t) \leq e_{q_{1} \vee q_{2}}(t)+e_{q_{1} \wedge q_{2}}(t)
$$

In particular,

$$
g_{q_{1}}+g_{q_{2}} \leq g_{q_{1} \vee q_{2}}+g_{q_{1} \wedge q_{2}}
$$

Consider $1-g_{q}$. We know that this function is excessive. Let

$$
C(q)=\mathscr{L}\left[1-g_{q}\right]
$$

where $\mathcal{L}$ is the mass-functional. (See [7].)

$C(q)$ has the following properties:

(A) $q_{1} \leq q_{2} \Rightarrow C\left(q_{1}\right) \leq C\left(q_{2}\right)$. Indeed, $g_{q_{2}} \leq g_{q_{1}} \Rightarrow 1-g_{q_{2}} \geq 1-g_{q_{1}}$.

(B) $C(q)<\infty \Rightarrow 1-g_{q}$ is a potential. (A harmonic function has infinite mass functional.)

(C) $C(0)=0$. This property is clear.

(D) $C$ is strongly subadditive, i.e., $C\left(q_{1} \vee q_{2}\right)+C\left(q_{1} \wedge q_{2}\right) \leq C\left(q_{1}\right)+C\left(q_{2}\right)$. This follows immediately from (5.3).

$(E)$

$$
\int\left|\nabla g_{q}\right|^{2}+\int q g_{q}^{2} \leq C(q)
$$

Proof: First, suppose that $q$ is integrable and $G q$ bounded. Then

$$
1-g_{q}=G\left(q g_{q}\right)
$$

holds. Thus $C(q)=\int q g_{1}$. Also, the potential $G\left(q g_{q}\right)$ has finite energy because $\int q g_{q} G\left(q g_{q}\right) \leq$ $\int q g_{q} \leq \int q<\infty$. The energy is $\int\left|\nabla G\left(q g_{q}\right)\right|^{2}=\int\left|\nabla\left(1-g_{q}\right)\right|^{2}=\int\left|\nabla g_{q}\right|^{2}$. After multiplying (5.4) by $q g_{q}$, integrating and using the above expression for $C(q)$, one gets

$$
\int\left|\nabla g_{q}\right|^{2}+\int q g_{q}^{2}=\int q g_{q}=C(q)
$$

Let $q_{n}$ satisfy $\int q_{n}<\infty$ and let $G q_{n}$ be bounded and let it increase to $q$. Then, $1-g_{q_{n}}$ increases to $1-g_{q}$. So, $C\left(q_{n}\right)$ increases to $C(q)$. If $C(q)=\infty$, the statement holds. Suppose $C(q)<\infty$. Then $\mathcal{L}\left[1-g_{q_{n}}\right]$ is bounded. Now, $1-g_{q_{n}} \in \mathfrak{H}_{0}^{1}(\Omega)$ and it is a bounded sequence in $\mathfrak{H}_{0}^{1}(\Omega)$. This sequence tends to $1-g_{q}$. Hence $1-g_{q} \in \mathfrak{H}_{0}^{1}(\Omega)$, and

$$
\int\left|\nabla\left(1-g_{q}\right)\right|^{2} \leq \underline{\lim } \int\left|\nabla\left(1-g_{q_{n}}\right)\right|^{2}
$$

Now $q_{n} \uparrow q, g_{q_{n}} \downarrow g_{q}$, so that $\underline{\lim }\left(q_{n}, g_{q_{n}}\right) \geq q g_{q}$, where the last is defined to be zero if $g_{q}=0$. So the 
result follows from Fatou's Lemma.

Theorem 5.1: If $s$ is excessive, then $G \overline{1} \not \equiv \infty$.

Proof: Let $x_{0} \in D$ and $B\left(x_{0}, r\right) \subset D$. For $y \notin B\left(x_{0}, r\right), G\left(x_{0}, \cdot\right)$ is harmonic in $D \backslash B\left(x_{0}, r\right)$ and continuous. So, for some $M, G\left(x_{0}, y\right) \leq M s(y)$ for all $y \in \partial B\left(x_{0}, r\right)$. Hence, $G\left(x_{0}, y\right) \leq$ $M s(y)$ for all $y \in D \backslash B\left(x_{0}, r\right)$. So,

$$
\int_{D \backslash B\left(x_{0}, r\right)} G\left(x_{0}, y\right) \frac{1}{s(y)} d y \leq M \cdot \lambda\left(D \backslash B\left(x_{0}, r\right)\right) .
$$

Here, $\lambda$ denotes the Lebesgue measure. In $B\left(x_{0}, r\right), s$ is bounded from below. So,

which completes the proof.

$$
\int_{B\left(x_{0}, r\right)} G\left(x_{0}, y\right) \frac{1}{s(y)} d y<\infty
$$

Thus,

$$
g_{s}=E^{x}\left[e^{-\int_{0}^{\tau} \frac{1}{s\left(X_{t}\right)} d t}\right]>0
$$

everywhere and satisfies $\Delta g_{s}=\frac{1}{s} g_{s}$.

\section{References}

[1] Aizenman, M. and Simon, B., Brownian motion and Harnack inequality for Schrödinger operators, Comm. Pure Appl. Math. 35 (1982), 209-273.

[2] Chung, K.L., On Stopped Feynman-Kac Functional, Séminaire de Probabilités XIV, Lecture Notes in Math. 784, Springer-Verlag, Berlin 1980.

[3] Chung, K.L. and Murali Rao, K., Sur la Theorie du Potential avec la Fonctionelle de Feynman-Kac, C.R. Acad. Sci., Paris 210 (1980).

[4] Chung, K.L. and Murali Rao, K, Functional and the Schrödinger equations, Seminar on Stochastic Processes, Birkhäuser, Boston (1981), 1-30.

[5] Dal Maso, G. and Mosco, U., Wiener criteria and energy decay for relaxed Dirichlet problems, Arch. Rational Mech. Anal. 95:4 (1986), 345-387.

[6] Kac, M., On some connections between probability theory and differential and integral equations, Proc. Second Berkeley Symposium on Math. Stat. and Probability, Univ. of California Press, Berkeley (1951), 189-215.

[7] Pop-Stojanović, Z.R. and Murali Rao, K., Convergence in energy, Z. Wahrsch. Geb. 69 (1985), 593-608. 


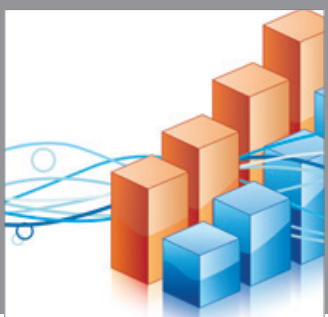

Advances in

Operations Research

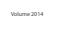

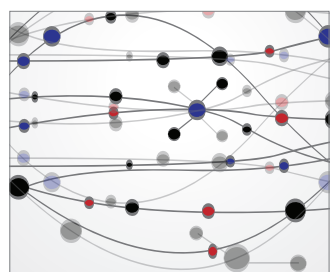

\section{The Scientific} World Journal
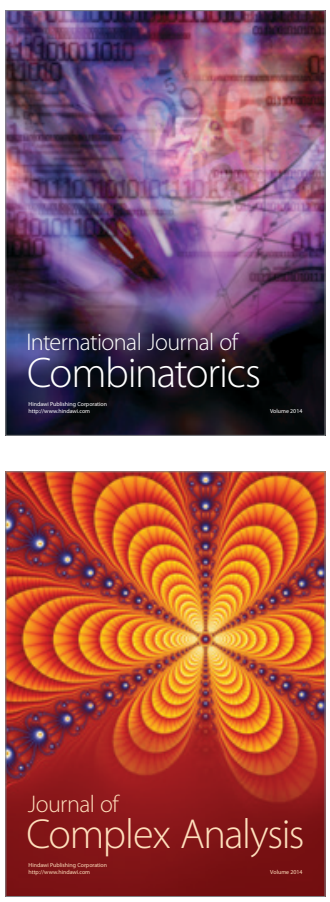

International Journal of

Mathematics and

Mathematical

Sciences
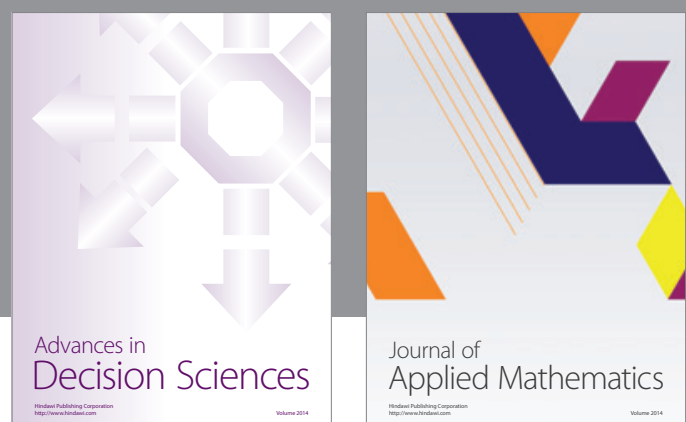

Journal of

Applied Mathematics
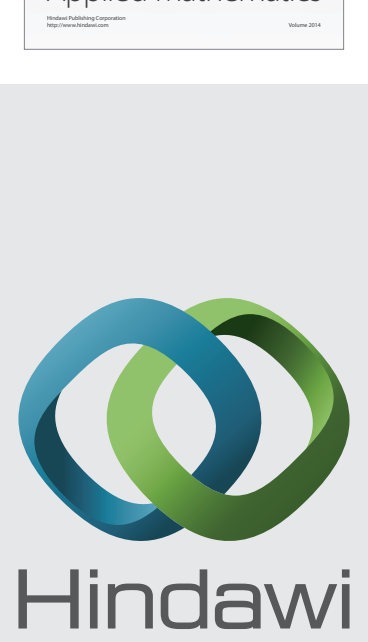

Submit your manuscripts at http://www.hindawi.com
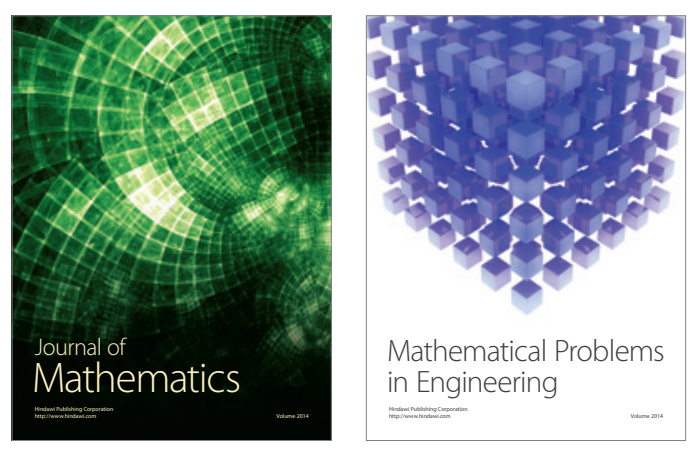

Mathematical Problems in Engineering
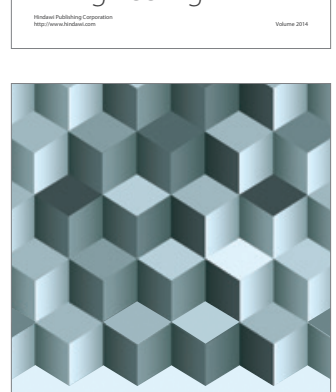

Journal of

Function Spaces
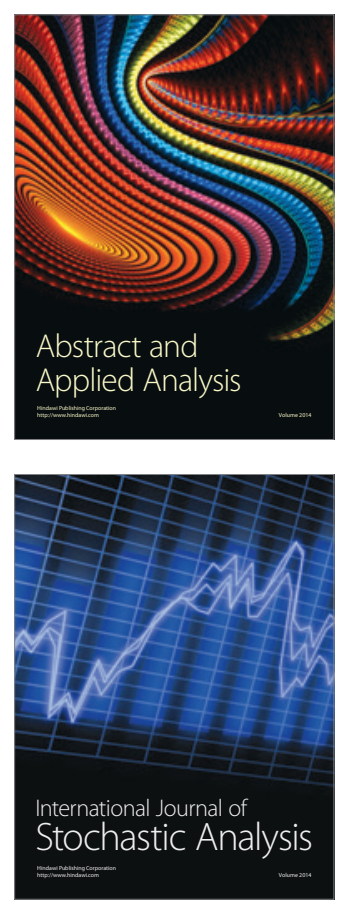

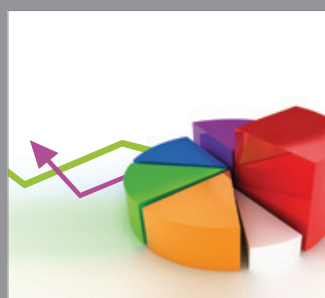

ournal of

Probability and Statistics

Promensencen
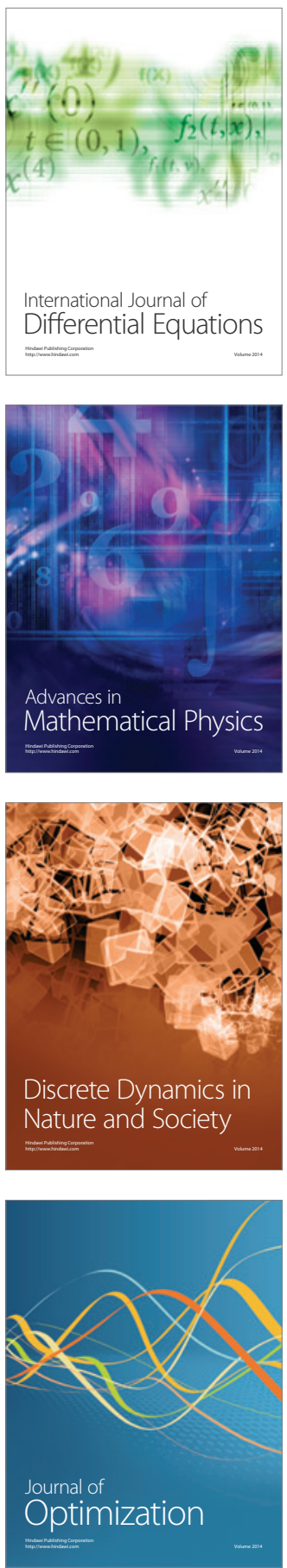